Editorial

\title{
Emergency Interventional Radiology
}

\author{
Shyamkumar N. Keshava ${ }^{1}$ Sanjeeva Kalva \\ ${ }^{1}$ Department of Radiology, Christian Medical College, Vellore, \\ Tamil Nadu, India \\ 2Division of Interventional Radiology, Department of Radiology, \\ Massachusetts General Hospital, Boston, Massachusetts, \\ United States
}

J Clin Interv Radiol ISVIR 2019;3:1-2

Interventional radiology has become an integral part of patient care services offered at many secondary and tertiary care hospitals in India. In addition to the routine elective consultation and procedural care for various vascular, nonvascular, and oncologic conditions, the interventional radiology service is being increasingly involved in the treatment of emergency conditions such as acute stroke or acute pulmonary embolism. The emergency interventional radiology service is more challenging compared with the elective service in several aspects, including, but not limited to, the need for quick decision-making given the clinical status of the patient, availability of resources for early intervention, and availability of alternative therapeutic options.

There are several factors that influence the outcome of emergency interventional treatment. These can be categorized into three groups: patient, material, and man power. The patient factors include the clinical status, ability to obtain the history and consent in a timely fashion, presence of a known diagnosis linked to the emergency problem, and associated comorbid conditions. It is important to assess the hemodynamic status of the patient and the pertinent laboratory parameters when an invasive procedure is planned. One has to be ready to make a balanced decision based on the clinical and imaging information already available versus obtaining more imaging tests to prevent further delay in offering a life-saving interventional therapy. If the clinical status allows, it may be prudent to obtain further information to aid the decision to perform a procedure or help steer the procedure in a particular direction. For example, in a stable patient with suspected acute gastrointestinal hemorrhage, it would be useful to know the site of hemorrhage on endoscopy or contrast-enhanced computed tomography prior to the patient being considered for embolization.

Material factors refer to the hardware for the procedure. The availability of appropriate devices (embolic materials, stent grafts, wires, catheters, etc.) is paramount in achieving an adequate therapeutic result. For example, a patient bleeding from a pseudoaneurysm requires an appropriately sized

Address for correspondence

Shyamkumar N. Keshava, MBBS, DMRD,

DNB, FRCR, FRANZCR, Department of

Radiology, Christian Medical College,

Vellore 632004, Tamil Nadu, India

(e-mail: aparna_shyam@cmcvellore.ac.in).

stent graft to exclude the aneurysm from the parent artery. Given the constrains of Indian hospitals to house multiple varieties of hardware for interventional procedures, it would be prudent to form a work group (consisting of hospital administrators, interventional radiologists, and industry representatives) within a city to mobilize the required material at a short notice.

Availability of personnel (man power) to provide care during the emergency is important and should be part of the hospital process improvement. The technologists, nurses, interventional radiologists, and, if required, the anesthesia team should be able to arrive at the hospital in an emergency within a relatively short time and short notice. Again, in our Indian scenario, it is important to work as a team of interventional radiologists at any given city to provide emergency coverage to multiple hospitals.

The American College of Radiology (ACR) has published practice parameters on clinical practice of interventional radiology. This is a collaborative document with the Society of Interventional Radiology (SIR), the Society of Neurointerventional Surgery (SNIS), and the Society for Pediatric Radiology (SPR). ${ }^{1}$ The document includes suggestions for the clinical team, patient services, equipment, quality improvement, radiation safety, etc. It has been recommended that the interventional radiologist be dedicated to the clinical management of patients in addition to performing the interventional procedures. Hospital admitting privileges have been mentioned as critical for a successful clinical interventional radiology practice. The Royal College of Radiologists has published "Standards for providing a 24-Hour Interventional Radiology Service."2 The document highlights the need for considering the safety of the patient first. One needs to be clear regarding the type of care provided. It also suggests discussing with strategic health care facilities and other hospitals to provide the service at any time. ${ }^{2}$. The availability and nonavailability of the type of emergency radiology services should be clearly mentioned, so that the patients are treated within the window period.

(C)2019 by Indian Society of

Vascular and Interventional Radiology

\section{License terms}

$10.1055 / \mathrm{s}-0039-1687197$

ISSN 2457-0214. 
Emergency as emergency, elective as elective. This summarizes that while it is important to identify a case as an emergency; it is equally important to identify what case is not when it is erroneously referred as an emergency. The latter would avoid misusing the emergency service and potentially compromising the patient care by offering the procedure suboptimally.

As interventional radiologists, we are committed to providing emergency interventional services. It is important that we, as a society, work together with the hospitals in laying guidelines for emergency interventional radiology service. This would not only improve the patient care and visibility of interventional radiology as one of the pillars of medicine but also enhance the appeal of interventional radiology as a specialty for future medical graduates.

\section{Conflict of Interest}

None declared.

\section{References}

1 ACR-SIR-SNIS-SPR. Practice parameter for interventional clinical practice and management. Available at: https://www.acr. org/-/media/ACR/Files/Practice-Parameters/IRClin-Prac-Mgmt.pdf. Accessed March 14, 2019

2 The Royal College of Radiologists. Standards for providing a 24-hour interventional radiology service. Available at: https://www.rcr.ac.uk/sites/default/files/docs/radiology/pdf/ Stand_24hr_IR_provision.pdf. Accessed March 14, 2019 